\title{
Non-contact geometry inspection of workpieces with optically non- cooperative surfaces
}

\author{
Friedrich-Wilhelm Bach ${ }^{1}$, Kai Möhwald ${ }^{1}$, Martin Nicolaus ${ }^{1, a}$, Eduard \\ Reithmeier $^{2}$, Markus Kästner ${ }^{2}$, Omar Abo-Namous ${ }^{2, \text { b }}$ \\ ${ }^{1}$ Leibniz Universität Hannover, Institute of Materials Science, D-30823 Garbsen, Germany \\ ${ }^{2}$ Leibniz Universität Hannover, Institute of Measurement and Automatic Control, \\ D-30167 Hannover, Germany \\ anicolaus@iw.uni-hannover.de, bomar.abo-namous@imr.uni-hannover.de
}

Keywords: optically non-cooperative surfaces, surface technology, surface metrology

\begin{abstract}
In order to produce optically cooperative surfaces to use triangulation measuring systems, the methods of surface and thin-coating technology are resorted to. Among these number physical methods such as PVD technology, (electro-)chemical treatment as, for example, electrochemical etching and electroless coatings as well as mechanical treatments of the components by means of glass-bead and corundum blasting. The processes mentioned are suitable for producing optically cooperative surfaces since, owing to the corresponding test parameters, a change of the component's surface occurs at the sub-micrometer scale. Currently, no indication in the literature is provided that such processes are employed for manufacturing optically cooperative surfaces vis-à-vis triangulation methods.
\end{abstract}

\section{Introduction}

The fringe-projection technique as an optical triangulation-procedure represents a rapid and flexible measuring method for recording areal geometries. For example, wear and shape defects of workpieces (e.g. gearwheels and synchronised discs) can be detected by using this technology. However, finished workpieces exhibit mainly very smooth and shiny surfaces which, owing to their reflecting behaviour, are not amenable to the fringe-projection technique [1]. Surface treatment methods represent one solution to this problem such as, for example, fine blasting or hydrodynamic abrasion processes with which the test piece's original mirror finish is roughened at the micrometer level and is thus rendered optically measurable. However, material removal using this process is so large that the shape of the workpiece is significantly influenced. The so-called thin-film processes are of great interest for treating the very shiny surfaces of components. Among these number physical methods such as PVD or CVD, (electro-) chemical processes, as well as mechanical treatments such as glass-bead and corundum blasting. Using these procedures, the material removal is clearly located in the sub-micrometer range and thus has practically no influence on the components shape. In addition to this, the material removal can be very accurately and flexibly controlled by means of the appropriate process parameters. An overview of the state of technology with regard to these processes can be found in [2].

In this work, technical surface treatments are carried out on stainless steel 1.4301 (X5CrNi18-10) and on case-hardening steel $1.7193(16 \mathrm{MnCrS5})$. The technical surface treatment's procedures for the case-hardening steel are interesting since this steel is employed for manufacturing gearwheels and synchronised discs and this procedure should be applicable for industrial quality control.

For evaluating the individual surface treatment methods, the roughness values of the specimens are both tactilely and optically measured and compared with the initial values. Tactile data was acquired using the measuring device; Perthometer PRK from the company Mahr. Optical testing was carried out by means of a light-scattering measurement system from the company Optosurf. The fringe projection system MikroCAD from the company GF Messtechnik is employed to verify 
the specimen's optical cooperativity regarding the measurement with triangulation techniques. Moreover, cylindrical specimens for assessing the radiant characteristics were measured using a measuring principle purposely developed for this task [1]. A further aim of the project is to attempt to correlate the tactile and optical surface roughness values.

Whenever there is talk of optical cooperativity within this paper, the optical cooperativity regarding the use of triangulation techniques such as fringe projection is meant.

\section{Experimental method}

For the surface treatment, polished cylindrical $(\varnothing: 20 \mathrm{~mm}, \mathrm{~L}: 20 \mathrm{~mm})$ and flat $(20 \mathrm{~mm} \times 20 \mathrm{~mm})$ specimens are employed. In preliminary tests for the feasibility study, stainless steel specimens were plasma-etched using PVD. The workpieces were etched in argon-plasma for $2.5 \mathrm{~h}$ at a HFpower of $700 \mathrm{~W}$. In other preliminary tests, specimens were electrochemically etched in diluted $\mathrm{H}_{2} \mathrm{SO}_{4}$ solution (20\%wt.). A simple galvanic cell was used for the electrochemical processing. The current was held constant by means of a potentiostat/galvanostat so that a current density of 1-2 $\mathrm{A} / \mathrm{dm}^{2}$ resulted. The workpiece was connected as the anode. An inert electrode was used as the cathode.

Industrial case-hardening steel 1.7193 (16MnCrS5) specimens of were used in further tests. Table 1 shows the selection of methods used here. As already mentioned above, mechanical, physical and electrochemical processes were employed.

Table 1:Selection of employed surface treatments

\begin{tabular}{|l|l|}
\hline \multicolumn{1}{|c|}{ Treatment methods } & \multicolumn{1}{c|}{ Parameter } \\
\hline blasting with fused alumina EKF1000 & $3-6$ bar blasting pressure, $5-20 \mathrm{~s}$ \\
\hline PVD etched with argon/nitrogen plasma & $2.5 \mathrm{~h}, \mathrm{HF}$ power $700-1400 \mathrm{~W}$ \\
\hline etched in $\mathrm{H}_{2} \mathrm{SO}_{4}(20 \% \mathrm{wt})$. & $1-15$ minutes \\
\hline electrochemically etched in $\mathrm{H}_{2} \mathrm{SO}_{4}(20 \% \mathrm{wt})$. & $1-5$ minutes, $1-2 \mathrm{~A} / \mathrm{dm}^{2}$ anodic \\
\hline electrochemically etched in $1-\mathrm{M} \mathrm{HCl}$ & $1-5$ minutes, $1-2 \mathrm{~A} / \mathrm{dm}^{2}$ anodic \\
\hline chemically copper-plated & $1 \mathrm{~h}$, various additives \\
\hline
\end{tabular}

For the chemical copper-plating, a standard copper electrolyte with different additives was employed which have an influence on the appearance of the copper coating.

Blasting with fused alumina EKF1000 having $5 \mu \mathrm{m}$ particles was selected for the mechanical methods. The setting parameters consisted of the jet distance from the object's surface, the equipment's blasting pressure ( 3 - 6 bar) and the blasting duration. In the initial tests, the blasting distance and duration was fixed at $10 \mathrm{~cm}$ and 5 seconds, respectively.

The roughnesses $\left(R_{a}\right.$ value) of the untreated and the treated specimens were tactilely measured and measured by using the fringe projection technique. It is initially assumed that the tactilely determined average roughness value Ra represents an indirect measure of the surface's optical cooperativity. However, this must be validated during the course of the test series. Furthermore, the behaviour of the specimen's optical light-beam was measured by means of both a simplified goniometric reflecting approach and also a scattered-light measuring device.

\section{Results}

Figure 1 shows the images of the untreated and the electrochemically etched specimen (20\% $\mathrm{H}_{2} \mathrm{SO}_{4}$ ), as well as the optically and tactually obtained roughness. 
$\mathrm{R}_{\mathrm{a}}$ (optical) $=5,4 \mu \mathrm{m}$

$\mathrm{R}_{\mathrm{a}}$ (tactile) $=0,8 \mu \mathrm{m}$
$\mathrm{R}_{\mathrm{a}}$ (optical) $=2,6 \mu \mathrm{m}$

$\mathrm{R}_{\mathrm{a}}($ tactile $)=0,9 \mu \mathrm{m}$
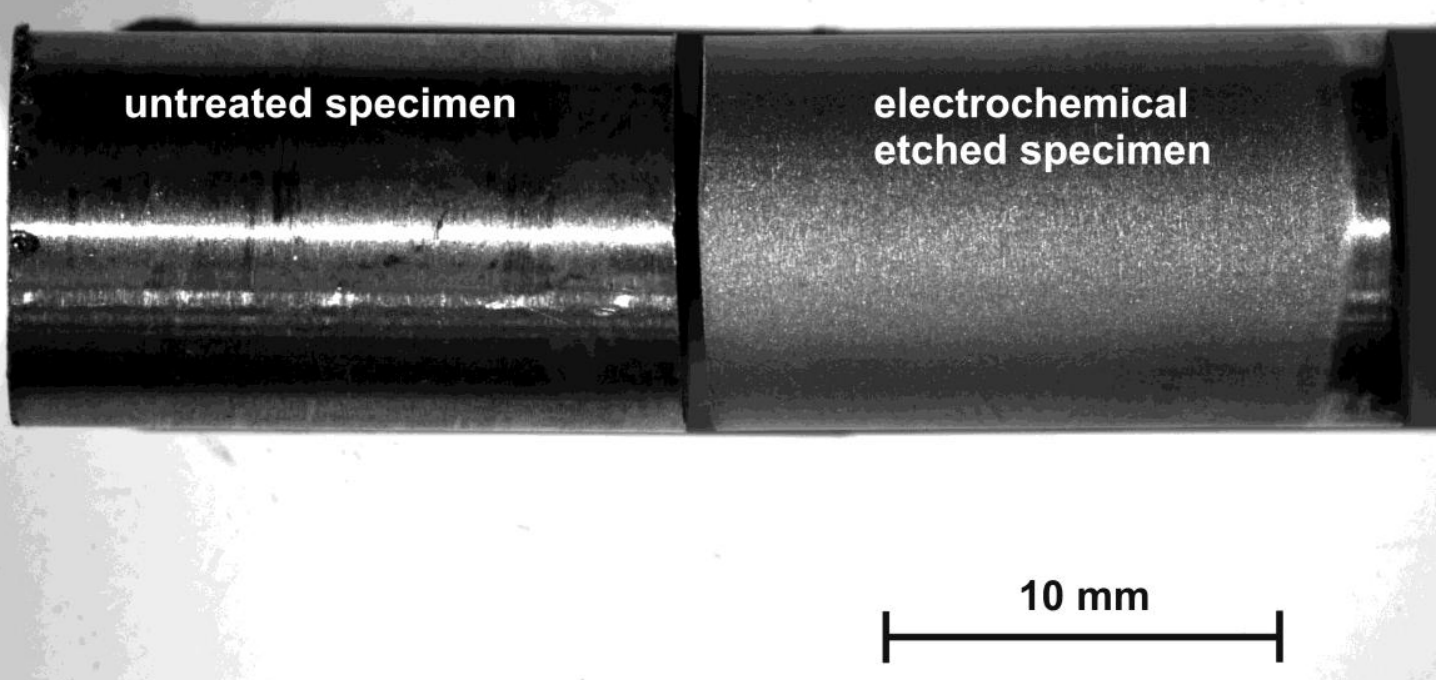

Fig. 1: untreated and electrochemically etched stainless steels

On the basis of figure 1, it can be seen that the untreated, shiny stainless steel specimen is highly reflecting and exhibits a high peak in the measure values whereas the electrochemically treated specimen is more optically cooperative and can be more homogenously illuminated. This behaviour is shown in the optically measured roughness value. The $\mathrm{R}_{\mathrm{a}}$ value for the untreated specimen is 5.4 $\mu \mathrm{m}$ and that for the treated specimen is $2.6 \mu \mathrm{m}$. The tactually determined roughness's for the untreated and treated specimens are $\mathrm{R}_{\mathrm{a}}=0.8 \mu \mathrm{m}$ and $\mathrm{R}_{\mathrm{a}}=0.9$, respectively.

The results of an untreated and flat specimen etched using argon-plasma shows similar characteristics (figure 2). The roughness's determined from the optically measured values provide an $R_{a}$ value of $14.4 \mu \mathrm{m}$ for the untreated stainless steel. surfaces, such as those exhibited by the plasma-etched stainless steel specimens, scatter the diffuse impinging light and are thus suitable for detecting by means of fringe projection: This is supported by the measured results obtained here. The roughness value $R_{a}$ determined from the fringe projection for the plasma-etched stainless steel is $2.7 \mu \mathrm{m}$. The tactilely determined $\mathrm{R}_{\mathrm{a}}$ value for the untreated specimen is $0.07 \mu \mathrm{m}$ and that for the plasma-etched specimen is $0.18 \mu \mathrm{m}$. 


$$
\begin{array}{ll}
\mathrm{R}_{\mathrm{a}} \text { (optical) }=14,4 \mu \mathrm{m} & \left.\mathrm{R}_{\mathrm{a}} \text { (optical }\right)=2,7 \mu \mathrm{m} \\
\mathrm{R}_{\mathrm{a}}(\text { tactile })=0,07 \mu \mathrm{m} & \left.\mathrm{R}_{\mathrm{a}} \text { (tactile }\right)=0,18 \mu \mathrm{m}
\end{array}
$$

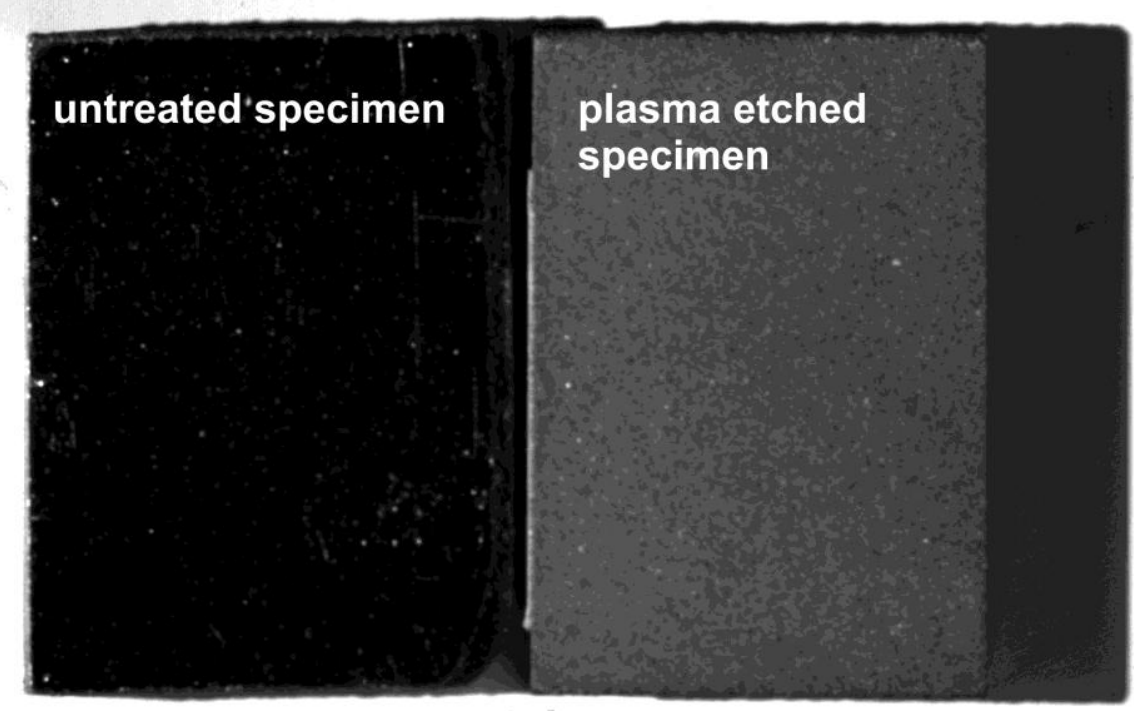

Fig. 2: untreated and plasma-etched stainless steel specimen

In regard to the optical cooperativity, the tests carried out here show that, in principle, it is possible to influence the surface characteristics by means of both physical as well as (electro-) chemical methods.

As previously mentioned, thin coating methods (table 1) are also used on the industrially employed case-hardening steel 1.7139.

The results for both the goniometric measurements using the scattered-light sensor Optosurf as well as the tactilely detected roughness values can be seen in figure 3 (diagram). The specimens are indicated on the abscissa. Specimens $1 p-4 p$ were sand-blasted with a blasting pressure of 2, 4, 6 and 8 bar respectively. Specimens $5 p-8 p$ were etched in a concentrated $\mathrm{H}_{2} \mathrm{SO}_{4}$ solution for $2,5,10$ and 15 minutes respectively. The specimens $9 p-11 p$ and $12 p-14 p$ were etched for 5,10 or 15 minutes in 0.1 mole $\mathrm{FeCl} 3$ solution and in citric acid, respectively. Specimens $15 \mathrm{p}$ and 16p were PVD-treated for 10 and 15, respectively. The values on the abscissa are the standard deviations of the scattering-cone modelled as a Gaussian curve. A higher value indicates a higher optical roughness. 


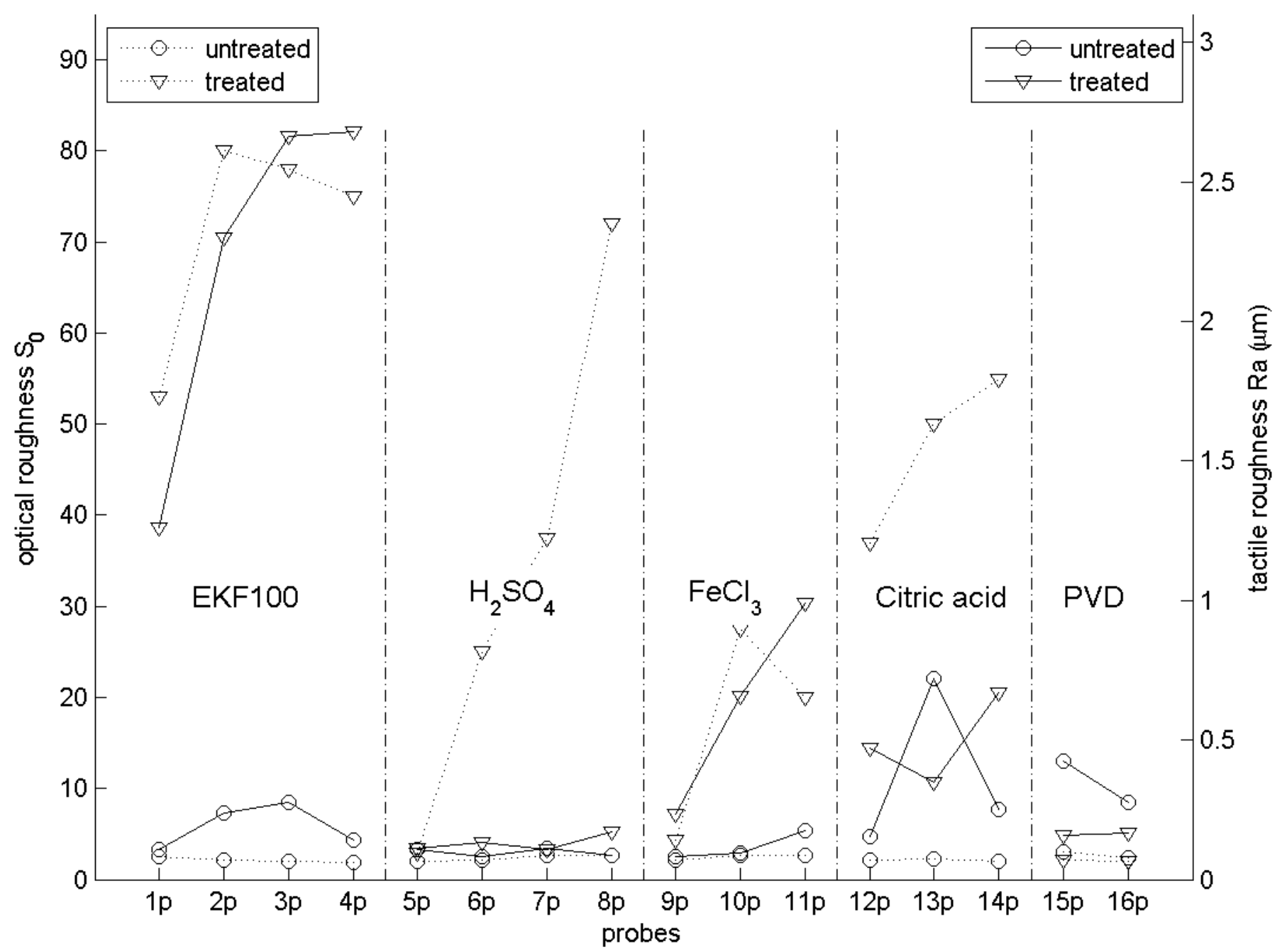

Fig. 3: tactile and optical specimen roughness values prior and subsequent to the surface treatment

All untreated specimens start at an approximate optical roughness value of about 2.5 and a tactile roughness of $0.2 \mu \mathrm{m}$. Except for the PVD-treated specimen all the treatment methods lead to a higher optical roughness. Having said that, the gonioreflectometric evaluation of the specimen's scattering characteristic in combination with the fringe projection measurements show that blasting with fused alumina provides exceptional results. Although the tactile roughness Ra remains within $3 \mu \mathrm{m}$, the optical roughness increases dramatically. The fringe projection measurement of specimen $4 \mathrm{p}$, which was half polished and half sand blasted using 8 bar, is shown in figure 4 . One clearly sees that using a fringe projection system the unprocessed half (upper part) can be measured only in a very narrow region around the shining effect, which means that the intensity contrast of the light reflected by the surface is too high for the dynamics of the camera chip. The surface treated bottom half of the specimen can be measured in a continuous manner over the entire visual field. This indicates a much higher optical cooperativity. 


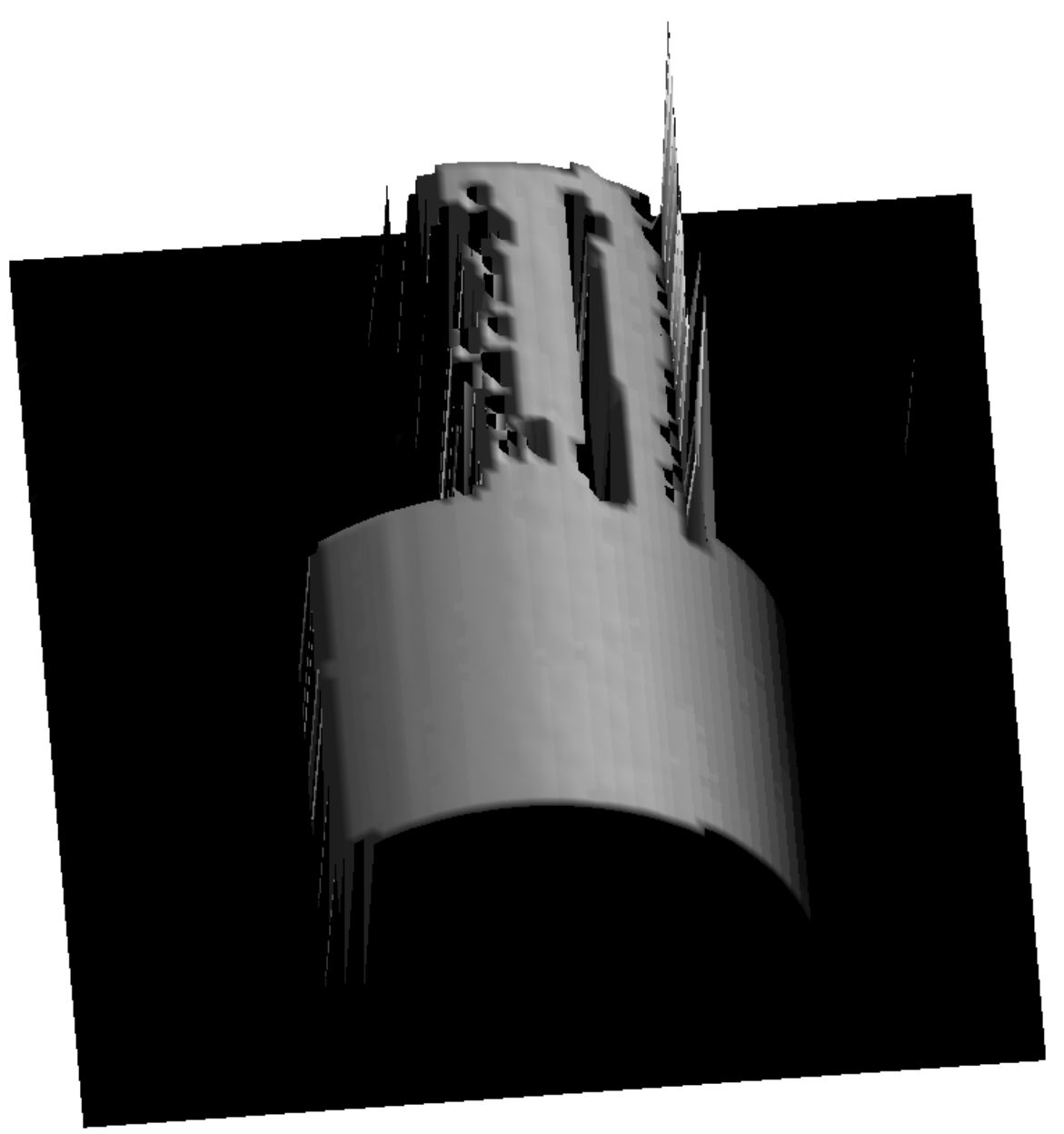

Fig. 4: test measurement of a partly sandblasted (lower part) probe (8 bar) using fringe projection

Also, electrochemical etching using 1-M HCl demonstrates, to some extent, promising results. The specimens treated in this way exhibit a wider area which can be measured using fringe projection techniques which can be substantiated using contact and optically determined roughness values.

\section{Conclusions}

On the basis of the tests carried out here, it is seen that it is, in principle, possible to produce optically cooperative surfaces by means of thin coating technologies in regards to triangulation measurement techniques. However, the methods to be employed must be appropriately matched to the materials to be measured.

In the course of the project, mainly the mechanical methods for surface treatment are to be more closely investigated.

\section{Acknowledgements}

The research is carried out within the scope of the transfer project T5 "geometry testing of optically incooperative surfaces” which is part of the collaborative research centre 489 „Process chain for the production of precision-forged high-performance parts". The authors would like to thank the German Research Foundation (DFG) for supporting and funding this research project. 


\section{References}

[1] Abo-Namous, O., Seewig, J., Kästner, M., Reithmeier, E. (2008): Fast method to measure optical cooperativity. Proc. DGaO, p. A16

[2] Nicolaus, M.; Schäpers, M.: Grundlagen der Dünnschichttechnologie; in: Bach, F.-W.; Möhwald, K.; Laarmann, A.; Wenz, T. (Hrsg.): Moderne Beschichtungsverfahren; WileyVCH Weinheim, 2004 